Archives

8 | 1991

Varia

\title{
Les revues
}

\section{Marie-Laurence Netter}

\section{OpenEdition \\ Journals}

Édition électronique

URL : http://journals.openedition.org/ccrh/2825

DOI : $10.4000 /$ ccrh. 2825

ISSN : 1760-7906

\section{Éditeur}

Centre de recherches historiques - EHESS

\section{Édition imprimée}

Date de publication : 15 octobre 1991

ISSN : 0990-9141

Référence électronique

Marie-Laurence Netter, «Les revues », Les Cahiers du Centre de Recherches Historiques [En ligne], 8|

1991, mis en ligne le 18 mars 2009, consulté le 10 décembre 2020. URL : http://

journals.openedition.org/ccrh/2825; DOI : https://doi.org/10.4000/ccrh.2825

Ce document a été généré automatiquement le 10 décembre 2020.

Article L.111-1 du Code de la propriété intellectuelle. 


\title{
Les revues
}

\author{
Marie-Laurence Netter
}

1 Entre le journal et le livre, la revue occupe une place intermédiaire au sens premier du terme parce que ces acteurs cherchent sciemment à concilier l'actualité de l'un et la profondeur d'analyse de l'autre. Le genre, pourtant, n'est pas hybride car il correspond à un besoin spécifique.

2 À l'aube $\mathrm{du} \mathrm{xx}^{\mathrm{e}}$ siècle, cette forme d'expression et de communication explose littéralement dans tous les domaines; fleurissent alors les revues en tout genre, techniques ou scientifiques, littéraires, intellectuelles ou militantes, promises à un avenir plus ou moins long, plus ou moins fameux. Toutes sont nées de la volonté d'un homme ou d'un petit groupe d'hommes animés de l'impérieuse nécessité de s'exprimer librement et de s'adresser à un groupe plus large. La revue, en ce sens, est le lieu par excellence de l'échange intellectuel puisque pensée comme tel.

3 L'objet de notre recherche étant précisément de mettre en lumière les mécanismes de la communication intellectuelle, on comprendra aisément que nous nous soyons attachés davantage au fonctionnement et à la diffusion des revues qu'à leur contenu. Pourquoi crée-t-on une revue, qui en prend l'initiative, qui la dirige? D'où viennent les fonds ? Quel est son rythme de parution et à quel prix est-elle vendue ? Combien a-t-elle d'abonnés ? A quel nombre d'exemplaires est-elle tirée, combien sont vendus? Enfin combien de temps dure cette revue? Nous avons essayé de répondre à ces questions très concrètes, parce que la vie matérielle d'une revue reflète assez objectivement, nous a-t-il semblé, le dessein qu'elle s'assigne et le succès rencontré, c'est à dire sa légitimité réelle. Par ailleurs elles conditionnent les réponses à une autre série de questions sur la qualité et la quantité des collaborateurs, occasionnels, réguliers, étrangers, etc., ce qui nous mène au coeur du problème dont nous voudrions traiter, à savoir la constitution de réseaux.

4 Nous avons examiné ainsi dix revues intellectuelles, plus que littéraires, politiques et syndicalistes $^{1}$. Par intellectuelles nous entendons des revues qui s'intéressent au mouvement des idées tant dans le domaine littéraire que dans le domaine artistique, politique et même scientifique. Cette ouverture correspond à un besoin fondamental, exprimé souvent clairement par les créateurs de revues eux-mêmes, d'indépendance et 
de liberté par rapport à une pensée ou un mouvement dont ils se sentent proches mais dont ils refusent les limites ou les impératifs doctrinaux. Ce besoin de liberté se double toujours, d'un besoin de reconnaissance. Les proches, amis ou relations, seront mis à contribution et constitueront avec les lecteurs, un double réseau où pourra s'épanouir et se fortifier une nouvelle façon de penser.

Quelque soit, en effet, la vocation dominante de la revue, ici culturelle ou syndicaliste, chaque fondateur privilégie l'aspect novateur de son entreprise par l'espace de liberté qu'il entend promouvoir. Les titres parlent parfois eux-mêmes de ce désir de s'affranchir de la contrainte d'une pensée trop structurée, c'est le cas de l'Indépendance (G. Sorel), de l'Effort libre (J.R. Bloch) ou encore de Pagine libere (Labriola). Dans tous les cas, le premier numéro est toujours l'occasion d'un manifeste ou d'un préambule qui permet de préciser les raisons de la création. C'est ainsi que Sorel déclare que l'Indépendance ne sera "pas l'instrument d'un parti » mais offrira, hors mode, l'occasion de se tenir au courant des grandes questions d'actualité et des meilleures productions littéraires d'un pays qui ne doit ni oublier ni renier ses traditions. Dans La Vie ouvrière, Monatte proclame « Nous n'avons ni catéchisme ni sermon à offrir ». Autour d'un « noyau » d'esprits libres qui ne partagent pas forcément les mêmes opinions, Monatte et ses amis veulent faire une revue différente, une revue d'action, sorte de coopérative intellectuelle où prévaudra la transparence financière. Avec la Revue socialiste, Malon veut diffuser un socialisme non dogmatique, sans «sectarisme » ni prétention à l'infaillibilité qu'il faut laisser au pape catholique» comme il l'écrivait plaisamment en janvier 1885. Enfin nul n'ignore la farouche volonté d'indépendance qui animait Péguy et le non-conformisme de ses Cahiers de la Quinzaine.

Le désir d'une démarche novatrice est naturellement à la base de toute création, à partir du moment où la revue créé n'est pas seulement l'instrument d'une idéologie ou le bulletin d'information d'un mouvement ou d'une institution. Ce qui est intéressant ici, c'est que les créateurs de revues ne veulent pas tant s'abstraire des mouvances politiques et syndicales dont ils sont proches que se débarrasser du langage dogmatique qui, selon eux, déjà, faisait des ravages. Tous ont des exigences de lisibilité, de sincérité... et de transparence financière. Péguy, qui voulait faire un « journal vrai »², en est l'archétype, de même que le fonctionnement des Cahiers est l'archétype des difficultés inhérentes à la formule.

7 La cause première de tous les maux est évidemment le problème du financement de la revue qui demande, au démarrage, une mise de fonds importante et ensuite d'être suffisamment vendue pour se suffire à elle-même.

La cause première de tous les maux est évidemment le problème du financement de la revue qui demande, au démarrage, une mise de fonds importante et ensuite d'être suffisamment vendue pour se suffire à elle-même. La seconde de ces conditions ne fut jamais complètement remplie, ce qui explique la courte existence de ces revues, tout au moins en conformité avec les ambitions initiales. Il faut maintenant s'intéresser de près à la vie matérielle de ces publications pour mieux saisir, a contrario, l'espèce d'utopie qui avait présidé à leur naissance. Il ne s'agit ici, en aucun cas, d'un jugement de valeur car, si le manque de réalisme des projets a largement contribué à la médiocre longévité des revues, leur réputation et leur influence ultérieures ne sont pas en cause, bien au contraire.

9 En l'absence d'archives, c'est dans les collections des revues elles-mêmes et dans les correspondances que nous avons cherché les réponses aux questions que nous nous 
étions posées. D'emblée il est clair que le personnage central de la revue est celui qui a voulu son existence, lui consacre l'essentiel de son temps et lui donne sa cohérence. Il s'agit de Malon pour la Revue socialiste, Monatte pour la Vie ouvrière, Sorel pour l'Indépendance, Leone pour Il Divenire sociale ou Prezzolini pour La Voce. Ces hommes ne sont pas propriétaire du titre mais ils en sont l'âme, et agissent en toute liberté en ce qui concerne son contenu. D'autres sont à la fois propriétaires et directeurs, il s'agit cette fois de Pelloutier pour L'Ouvrier des deux mondes, Lagardelle pour le Mouvement socialiste, Péguy pour les Cahiers de la Quinzaine, J.R. Bloch pour l'Effort libre. Mais quelque soit leur fonction officielle, tous s'occupent en réalité de tout : d'abord ils écrivent eux-mêmes des articles et rédigent parfois l'essentiel de la revue. C'est le cas de Malon, Sorel, Bloch et bien sûr de Péguy puisqu'à la fin les Cahiers seront exclusivement son oeuvre. Tous veillent à la qualité des écrits qu'ils publient mais ils remplissent aussi, peu ou prou, les fonctions de secrétaire et d'administrateur en s'occupant personnellement de l'impression, de la diffusion et en recherchant sans cesse de nouveaux abonnés.

10 Leur dynamisme est véritablement l'épicentre de la vie de la revue qui, sans eux, n'existerait pas. Cela est si vrai que, sauf exception ${ }^{3}$, aucune de ces publications n'a survécu au désengagement de son créateur et nous touchons là aux limites de l'entreprise. En effet, ce désengagement est toujours dû à des difficultés financières, ellesmêmes dues à un trop petit nombre de ventes et notamment des abonnements; engrenage qui entraîne d'abord une transformation de la revue puis sa disparition. C'est à dire que la ligne choisie, celle de l'autonomie de pensée, ne fait pas recette. L'exemple de l'Ouvrier des deux mondes est tout à fait caractéristique.

11 Le petit mensuel de 16 pages publié par Pelloutier avait pour objectif de donner aux ouvriers des informations, mais aussi des études sur la vie économique et sociale qui auraient pu leur permettre de se forger une culture propre. La publication ne dure que deux ans et demi et Pelloutier jette l'éponge en juillet 1899. Il est déçu par le manque de sérieux des ouvriers français qui ne lisent pas suffisamment, et plus encore, rendu furieux par le verbalisme qui a envahi la revue depuis qu'elle a été rachetée par la Fédération des bourses du Travail et qu'elle doit publier des comptes rendus. Quoique l'expérience ait été particulièrement courte, son influence sera grande sur les publications ultérieures, au sein du mouvement ouvrier, notamment en ce qui concerne la rigueur de l'information.

La longévité moyenne de ces revues est plutôt de cinq ans, même si quelques unes ont réussi à paraitre plus longtemps. Mais, au fond, peu importe la durée puisque toutes se heurtent aux mêmes difficultés et cherchent, finalement, à les résoudre avec les mêmes expédients.

13 Le rythme de parution de ces revues est mensuel ou bimensuel et, généralement, soumis à variations. L'Indépendance, par exemple, est bimensuel jusqu'en août 1912, puis mensuel jusqu'en mai 1913 et à nouveau bimensuel jusqu'en juillet 1913, date de sa disparition. Chacun de ces changements correspond à une étape importante de la vie de la revue : en août 1912, il s'agit d'un remaniement du comité de rédaction qui prend une coloration nettement nationaliste avec l'arrivée de Maurice Barrès et de Paul Bourget. En mai 1913, Sorel vient de quitter complètement la rédaction et la revue devient un petit brûlot hyper nationaliste, promis à une fin prochaine.

14 Le tirage et le nombre des abonnés sont rarement connus avec précision mais, lorsqu'ils le sont, on constate qu'ils manifestent une nette tendance à la fluctuation. L'Ouvrier des deux mondes est d'abord tiré à 2.500 exemplaires puis à 2.800 et chute ensuite à 1.000 , traduisant en chiffres le découragement de Pelloutier. La vie ouvrière a de 1.600 à 1.800 
abonnés, alors qu'il lui en faudrait 4.000 pour vivre sans soucis ${ }^{4}$; l'Effort libre ne dépasse pas les 450 , alors qu'il lui en faudrait 800 d'après J.R. Bloch.

Les problèmes matériels obligent les directeurs de revues à rechercher de nouveaux lecteurs et, pour ce faire, ils infléchissent la ligne initiale. L'expérience montre qu'ils perdent alors sur les deux tableaux, les premiers abonnés cessent de les suivre mais les nouveaux n'affluent pas pour autant.

Si l'influence de ces revues fut donc manifestement modeste au sein du public, ouvrier ou lettré, auquel elles s'adressaient, leur notoriété ultérieure justifie, a posteriori, la pertinence de l'entreprise. La personnalité de leur créateur en est, sans aucun doute, la cause majeure mais l'originalité de la démarche, la variété et la qualité de leurs collaborateurs en est une autre d'importance. Les auteurs - écrivains, artistes, hommes politiques, philosophes - conviés à donner un ou plusieurs articles à ces différentes revues sont extrêmement nombreux et forment de véritables réseaux de dimension nationale et parfois internationale. Le Mouvement socialiste, en quinze années d'existence, a compté 544 collaborateurs de nationalités diverses et aux noms souvent prestigieux: Bernstein, Rosa Luxembourg, Labriola, Delesalle, Jaurès, Daniel Halévy, Sorel, Croce, Miches et tant d'autres, ont écrit, plus ou moins régulièrement pour la revue de Lagardelle. Certains de ces hommes écrivent aussi pour la Revue socialiste à laquelle Péguy collabora avant de fonder les Cahiers. Romain Rolland, les frères Tharaud, Claudel ont publié des textes dans l'Indépendance et dans les Cahiers de la Quinzaine...

On pourrait multiplier les exemples de ces collaborations croisées qui tissent, en fait, un seul et vaste réseau d'hommes, et quelquefois de femmes, venus de tous les horizons. Les uns entretiennent de bonnes relations, les autres s'ignorent ou feignent de s'ignorer mais tous apprécient la liberté d'expression que leur permet ce type de revues, indépendantes de toute institution, et c'est sans doute là leur grand succès.

Finalement, ces revues n'ont pas créé de réseau intellectuel très nouveau, et sur le plan de la diffusion elles ont échoué, mais elles ont su attirer à leurs débuts, des collaborateurs brillants qui ont assis pour longtemps leur renommée. Une renommé qui, dans tous les cas, est identifiée à un homme tant il est vrai, comme nous venons de le voir, qu'une revue est d'abord l'oeuvre de son créateur qui lui donne son temps, éventuellement son argent, mais toujours son âme.

\section{NOTES}

1. M. REBERIOUX, « La Revue socialiste »; J. JULLIARD, «L'ouvrier des deux mondes »; M. de FLERS, «Le Mouvement socialiste (1899-1914)»; G. LEROY, "Les Cahiers de la Quinzaine»; C. CHAMBELLAND, « La Vie Ouvrière (1909-1914)»; M.L. NETTER, « Georges Sorel et l'Indépendance »; C. PROCHASSON, "L'Effort libre de Jean-Richard Bloch (1910-1914»; W. GIANINAZZI, «Il Divenire sociale et Pagine libere»; B. SOMALVICO, "La Voce et la reformulation de l'identité culturelle italienne » : dans Cahiers Georges Sorel, $n^{\circ}$ 5, 1987.

2. L'expression est de Péguy lui-même, dans la Revue socialiste, janvier 1898, et G. LEROY, op. cit., p. 77. 
3. Il s'agit de la Revue socialiste créée par Malon en 1885 et qui existe encore aujourd'hui, après diverses interruptions et renaissances. Mais après la mort de Malon en 1893, la revue se transforme et se politise nettement à partir de 1900.

4. La Vie ouvrière, $\mathrm{n}^{\circ} 1,5$ octobre 1909.

\section{AUTEUR}

\section{MARIE-LAURENCE NETTER}

Marie-Laurence NETTER est ingénieur d'études à l'EHESS et membre du Centre de Recherches Historiques. 